\title{
Management of injuries in snowboarders: rehabilitation and return to activity
}

This article was published in the following Dove Press journal: Open Access Journal of Sports Medicine

\section{Kathryn Helmig \\ Gehron Treme \\ Dustin Richter \\ Department of Orthopaedic Surgery, University of New Mexico, Albuquerque, NM, USA}

Correspondence: Dustin Richter Department of Orthopaedic Surgery, University of New Mexico - MSC IO 5600 , I University of New Mexico, Albuquerque, NM 87I3I, USA

Tel +I 5052724107

Fax + I 5052728098

Email DLRichter@salud.unm.edu

\begin{abstract}
Snowboarding has seen a continuous increase in popularity, leading to an increase in the number of snowboarding injuries seen in orthopedic practice. Upper-extremity injuries are more common than lower-extremity, spine, and pelvis injuries. In this review, we focus on the most common snowboarding injuries of the extremities, spine, and pelvis and provide an overview of their respective rehabilitation and return-to-sport protocols. Despite many of the injuries seen in snowboarding also occurring in other sports, objective data about rehabilitation and return to sport are lacking for many injuries. This provides an opportunity for research in the area with regard to many sports and many different injuries.
\end{abstract}

Keywords: snow sports, return to sport, physical therapy, rehab, wrist injuries, lower extremity, spine, pelvis

\section{Introduction}

No longer simply an alternative to skiing, snowboarding has increased continuously in popularity over the last several decades. An estimated 8.2 million people participated in snowboarding in 2010 in the US. ${ }^{2}$ The increase in participation, along with the advent of terrain parks, which are the site of about $20 \%$ of injuries, has resulted in an increasing number of snowboarding injuries seen in orthopedic practice. Since 2001, injuries among snowboarders have occurred at a higher rate than those among skiers, with falls causing $80 \%-90 \%$ of injuries. ${ }^{2,3}$ Upper-extremity injuries are more common than those of the lower extremity, particularly among recreational snowboarders. Wrist injuries accounted for $27.6 \%$ of all injuries in one study, as snowboarders typically maintain balance with outstretched arms and use their upper extremities to brace themselves when they fall. ${ }^{2,4}$ In addition, it has been found that inexperienced, young, female riders are at greatest risk of injury. ${ }^{2}$ Lower-extremity injuries are decreasing in incidence with advances in boot and binding technology. ${ }^{1}$ Not only does snowboarding result in injury patterns unique to the sport, but the presence of snowboarders on the mountain has also been shown to increase overall injury rates to all snow-sport participants. ${ }^{5}$ Head injuries are also a significant cause of injury and disability among snowboarders. However, head injuries will not be addressed in this review, as they have already been discussed in this journal. ${ }^{6,7}$ In this review, we discuss the most common snowboarding injuries of the extremities, spine, and pelvis. While rehabilitation protocols and return-to-sport measures have not been explicitly defined for snowboarding for many injuries, we discuss general objectives and guidelines for return to sport after treatment. 


\section{Upper extremity}

The upper extremities are the most common site of injury among recreational snowboarders. ${ }^{2}$ Upper-extremity injury in snowboarding ranges from ligamentous sprains to fractures and from the hand distally to the shoulder and clavicle proximally. In a study evaluating 7,430 upper-extremity injuries over ten seasons at Colorado resorts, Idzikowski et al found that just under half of all snowboarding-related injuries involved the upper extremities, with male riders accounting for $74 \%$ of all injuries. ${ }^{8}$ Upper-extremity injuries included fractures $(56.4 \%)$, sprains $(26.8 \%)$, and dislocations $(9.7 \%){ }^{8}$ They found that wrist injuries made up $44 \%$ of all upperextremity injuries and $21.6 \%$ of all snowboarding injuries. ${ }^{8}$ As the upper extremities play a lesser role in comparison to the lower in snowboarding, general themes for return after these injuries include full functional range of motion and strength so that athletes can protect themselves and minimize additional risk.

\section{Distal radius fracture}

The most common upper-extremity snowboarding-injury location is the wrist, and distal radius fractures (Figure 1) are the most common wrist injury, nearly always resulting from a fall backward while riding. ${ }^{8}$ In one study, nearly $78 \%$ of wrist injuries were fractures and half of all distal radius fractures occurred in the 10- to 19-year-old age-group. ${ }^{8}$ With the exception of the scaphoid, fractures about the wrist were found most commonly in women, young riders, and beginners. ${ }^{8}$ Those riders wearing wrist guards were half as likely to sustain wrist injuries as those without protection. ${ }^{8}$

If treated nonoperatively, the patient is immobilized in a cast or splint for 4-6 weeks. Range-of-motion exercises

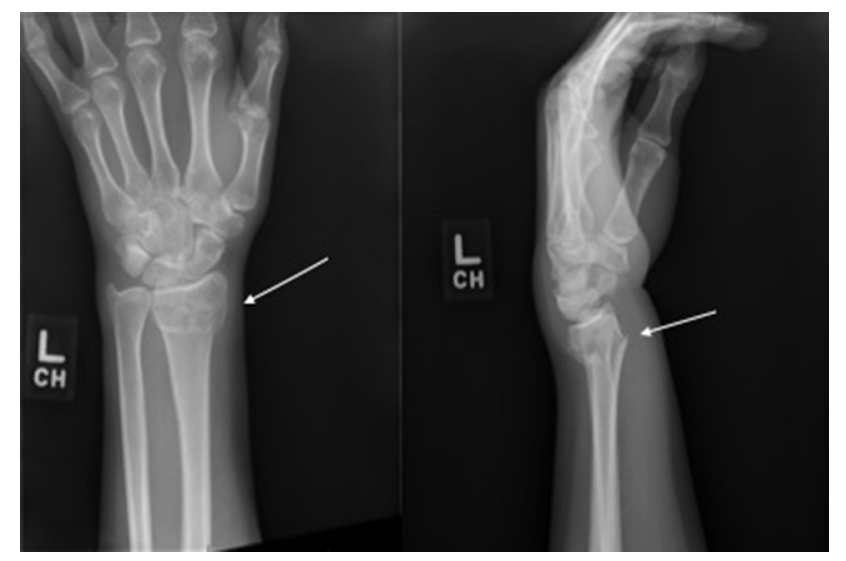

Figure I Distal radius fracture (white arrows). for the unaffected joints (fingers, elbow, and shoulder) may begin immediately. ${ }^{9}$ Once the period of wrist immobilization is completed, the patient should begin gentle active and passive wrist range of motion. Wrist-strengthening exercises should be withheld until radiographic evidence of union is present and the fracture site untender to palpation, typically around 6 weeks. The athlete may then begin strengthening exercises of the wrist and hand and be assessed every 1-2 weeks for consideration of return to sport. ${ }^{9}$ Strengthening exercises should begin with activities of daily living and progress to sport-specific strengthening. Return to sport for nonoperatively treated distal radius fractures is generally based on painless, functional range of motion of the wrist and ability to use the affected hand without guarding, which typically is at 6-9 weeks after injury. ${ }^{9}$

Operatively treated distal radius fractures are typically stabilized after surgery with an irremovable splint for 10-14 days, followed by 4 weeks in a removable brace. ${ }^{9}$ As with nonoperative treatment, range-of-motion exercises for the unaffected joints should be started immediately. Beleckas and Calfee recommended active wrist range of motion beginning at 2-6 weeks, depending on fracture severity. ${ }^{9} \mathrm{At}$ 6 weeks or when radiographic union is noted, passive wrist range of motion should begin and the brace discontinued. ${ }^{9}$ The athlete may also begin strengthening exercises at 6 weeks or with evidence of union on X-ray. Most athletes regain the majority of motion and function in the first 3 months of rehabilitation, but often continue to have improvements for at least 1 year. As with nonoperatively treated distal radius fractures, return to sport typically occurs at 6-9 weeks. Henn and Wolfe recommended radiographic healing and at least $80 \%$ return to motion and strength prior to return to sport. ${ }^{10}$ Beleckas and Calfee discussed returning to snowboarding in a cast prior to fracture healing, with the athlete's understanding that a new fall or collision may result in further injury. ${ }^{9}$

\section{Scaphoid, perilunate, and ligamentous wrist injury}

Isolated ligamentous wrist injury and injuries of the scaphoid and perilunate complex of the wrist in snowboarding typically occur in advanced riders, as they are associated with highenergy collisions. ${ }^{8}$ They have been noted to occur more frequently in males, and are often preceded by an aerial maneuver and result from a backward fall. Though far less common than distal radius fractures, these injuries can result in lost time participating in snowboarding activities and dysfunction, particularly if not recognized and treated early. 
After a ligament injury, the wrist is generally immobilized for 4-6 weeks. ${ }^{11}$ For elite athletes in season, one can consider return to sport with the use of a wrist splint or cast. During wrist immobilization, the patient should begin passive and active range-of-motion exercises of the unaffected upper-extremity joints, in order to limit stiffness and deconditioning. Once the period of immobilization is complete and pain minimal, the patient can begin strengthening and proprioceptive exercises of the wrist. In instances of acute, unstable ligamentous injuries, operative management may be indicated. ${ }^{11}$ If so, rehabilitation afterward mimics that of nonoperative treatment, with a period of immobilization followed by wrist strengthening and proprioceptive training. Return to sport varies widely for ligamentous wrist injuries. Athletes with minor injuries may return to sport almost immediately in a cast or splint. For more severe injuries, return to sport may not occur for 4-6 months. ${ }^{11}$ Timing of return to sport without a cast or splint may be assessed with full, painless range of motion and no tenderness to palpation. Typically, snowboarding is a sport in which return to activity with a cast or splint is acceptable.

Whether treated operatively or nonoperatively, scaphoidfracture rehabilitation first involves a period of immobilization in a splint or cast. ${ }^{12}$ Active and passive range of motion of the uninvolved joints is encouraged during this time. At week 6 for operatively treated fractures and 10-12 weeks for nonoperatively treated fractures, the patient can begin gentle active range of motion of the wrist and thumb. ${ }^{12,13}$ At 10-12 weeks, athletes should begin grip strengthening and advance as tolerated to progressive resistive exercises. ${ }^{12,13} \mathrm{~A}$ wrist splint may be used for protection after removal of the cast in both groups. ${ }^{12,13}$ At 3 months, the patient may return to full activity and sport if they are pain free. ${ }^{13}$ Symes and Stothard reviewed treatment of acute scaphoid fractures and found that all studies included in their review showed faster return to function after surgical treatment of scaphoid fractures vs cast immobilization alone. ${ }^{14}$

For lunate and perilunate dislocations, operative intervention is the standard of care. ${ }^{15}$ The patient is immobilized in a thumb-spica cast for 3-6 weeks postoperatively, and then active-assisted and gentle passive motion may begin. ${ }^{15,16}$ The athlete may wear a splint as needed for support once the thumb-spica cast is removed, but dynamic splints should not be used. ${ }^{15}$ At 3 months, grip-strength exercises and use of the wrist in activities of daily living begins. ${ }^{16}$ At 4-6 months, the athlete may return to sport if they are pain-free with good range of motion and strength. ${ }^{16}$ Commonly, for this injury, 12 months of continuous reha- bilitation is required before athletes have enough function and strength to return to sport. ${ }^{16}$

\section{Thumb ulnar collateral ligament injury}

Thumb ulnar collateral ligament injuries are more commonly seen in skiers, but can also be seen in snowboarders, particularly with falls. Riders treated nonoperatively may return to sport immediately with immobilization of the thumb in a thumb-spica cast or thermoplastic splint for at least 4 weeks. ${ }^{13}$ Patients who undergo operative repair of the ligament for an unstable injury should not return to sport until the wound has healed. ${ }^{17}$ The patient may then return with a splint or cast, which Goldfarb et al recommended be worn for a total duration of 12 weeks, while others have recommended immobilization for a total of 4-6 weeks. ${ }^{13,17}$ The period of sport with cast immobilization should ultimately be decided by severity of injury and by the operating surgeon. Regardless of treatment and timing of return, the thumb should be protected while snowboarding until the patient has full functional motion and no pain. Snowboarders should expect to remain in some form of protection for at least the duration of the season during which these injuries occur.

\section{Humerus fracture}

Diaphyseal and proximal humerus fractures are the two most commonly occurring humerus fractures in snowboarders. ${ }^{18}$ Bissell et al studied humerus fractures at a mountain clinic, and found that $71 \%$ of humerus fractures in snowboarders were left-sided, as the majority of snowboarders lead with their left foot. ${ }^{18}$ Almost a third of humerus fractures in the study were secondary to jumps. ${ }^{18}$ Dry powder, packed powder, lower skill level, and younger age were other risk factors for humerus fracture. ${ }^{18}$

Proximal humerus fractures are typically initially immobilized in a cuff-and-collar or shoulder immobilizer. Whether treated operatively or nonoperatively, the patient may begin finger and wrist range of motion immediately, as well as shoulder pendulums. ${ }^{19}$ Patients treated operatively should avoid more than $90^{\circ}$ of shoulder abduction and active range of motion for 6 weeks postoperatively. ${ }^{19}$ At 6 weeks, whether treated operatively or nonoperatively, a repeat X-ray is taken, and if radiographic healing is present, the patient may begin unrestricted range-of-motion exercises. At 10 weeks, patients may begin shoulder strengthening and scapular stabilization. Resistance exercises may begin at 12 weeks, and gradually increase to sport-specific exercises. Return to sport typically occurs at 3-6 months. ${ }^{19}$ 
Use of the extremity is allowed immediately with diaphyseal humerus fractures, with restrictions on lifting, weightbearing, and shoulder abduction. As soon as tolerated, the athlete may use the hand and forearm and begin shoulder pendulum exercises, elbow flexion, and elbow extension. Patients treated with an intramedullary nail may begin partial weight-bearing on the affected arm 4 weeks after surgery. ${ }^{20}$ Weight-bearing and strengthening exercises can be advanced once callus formation is present on radiography. This is also true for fractures treated with plate-and-screw fixation. ${ }^{20}$ For both types of fixation, scapular stabilization exercises may start at 8 weeks and resistance strengthening at 10 weeks. The progression of exercises should be gradual and as tolerated by the patient. Sport-specific exercises can typically begin around 4 months, with return to sport ultimately being between 6 months and 1 year.

Whether treated operatively or nonoperatively, distal humerus fractures are initially immobilized in a sling for comfort with the elbow at $90^{\circ}$ of flexion..$^{21}$ Finger range of motion can begin immediately, as well as shoulder pendulum exercises in the sling. Active shoulder abduction and resultant varus elbow stress should be avoided for the first 6 weeks. Passive- and active-assisted range of motion of the elbow can begin at 7-14 days to prevent elbow stiffness. ${ }^{21}$ The athlete should not begin weight-bearing or strengthening exercises until 6-8 weeks or until radiographic healing is evident. ${ }^{21}$ Progression of exercises should be gradual and performed as tolerated, with a typical return to sport around 6 months to 1 year.

\section{Clavicle fracture}

Clavicle fractures (Figure 2) in snowboarders account for around $5 \%$ of all snowboarding injuries, but are increasing with the increasing incidence of terrain parks: ${ }^{22} 34 \%$ of clavicle fractures in snowboarders are a result of a jump, loss of control, and impact with the snow's surface. ${ }^{22} \mathrm{Kim}$ et al examined data from a clinic near a popular ski area, and found that $94 \%$ of all clavicle fractures secondary to snowboarding occurred in males and $44 \%$ in terrain parks. ${ }^{22}$ The majority of clavicle fractures $(85 \%)$ were sustained by snowboarders who were intermediate-skill level or higher. ${ }^{22}$

Whether treated nonoperatively or operatively, the affected upper extremity is usually immobilized in a sling for 2-4 weeks, with range-of-motion exercises as tolerated. At 6 weeks, strengthening exercises of the shoulder and upper extremity may begin. In patients with undisplaced or very minimally displaced fractures, return to sport is typically at 10 weeks. ${ }^{23}$ Return to sport occurs at 21 weeks on average for displaced midshaft clavicle fractures managed conservatively and 9 weeks for displaced midshaft clavicle fractures treated surgically. ${ }^{23,24}$ Patients with distal clavicle fractures typically return to sport around 15 weeks when managed conservatively and 20 weeks when managed surgically. ${ }^{23}$ Guidelines for return to sport are complete, painless range of motion of the shoulder and full strength at the shoulder, particularly in flexion and abduction. ${ }^{23}$

\section{Acromioclavicular joint injury}

Acromioclavicular (AC) joint injuries (Figure 3) are more common among advanced riders, given the high speed typically needed to cause the injury. ${ }^{4}$ Ogawa et al examined the incidence of $\mathrm{AC}$ dislocation over five snow seasons retrospectively, and determined an incidence of $17 \% .{ }^{25} \mathrm{AC}$ injuries are typically immobilized for 2-4 weeks. ${ }^{26}$ Range of motion of the fingers, wrist, forearm, and elbow can begin immediately. After the period of immobilization, rehabilitation

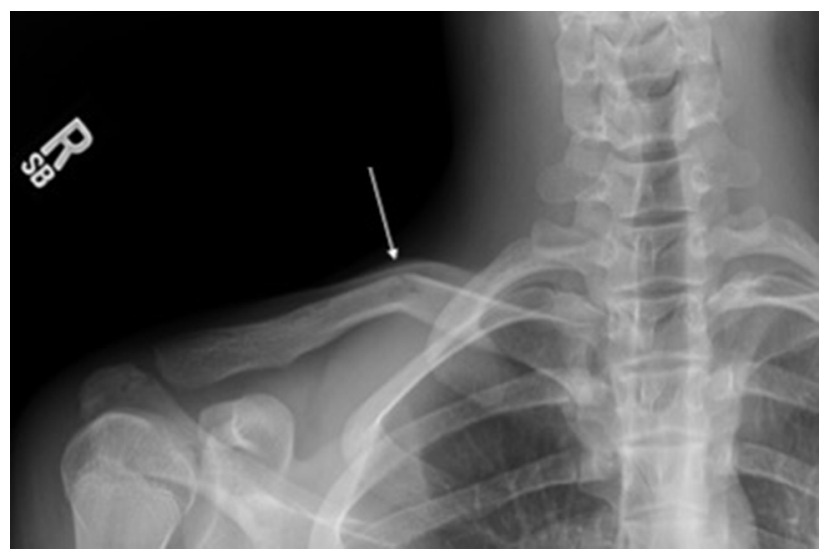

Figure 2 Clavicle fracture (white arrow).

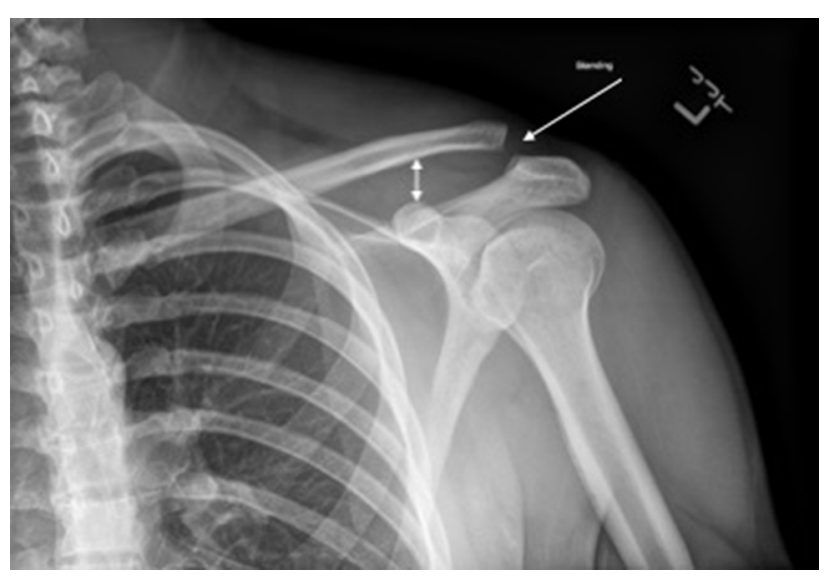

Figure 3 Acromioclavicular injury (white arrows). 
should include range of motion and strengthening at the shoulder. ${ }^{26}$ Return-to-play criteria following an AC joint injury are described as painless motion at the shoulder, full shoulder strength with flexion and horizontal adduction/abduction, and the ability to perform pain-free shoulder presses and bench presses. ${ }^{26}$ This typically occurs at a minimum of 6 weeks from the time of injury. ${ }^{26}$

\section{Shoulder dislocation}

The glenohumeral joint is the most common site of dislocation among snowboarders, accounting for $50 \%$ of dislocations in a large retrospective study by Ogawa et al. ${ }^{25}$ Almost $70 \%$ of glenohumeral dislocations occur with falls forward or to the toe side of the board, and $66 \%$ occur on the left side with a regular stance. ${ }^{27}$ Ogawa et al found risk factors for shoulder dislocation in snowboarding to be male sex, older riders, wet-snow conditions, and falls. ${ }^{25}$ Factors that were not found to play a statistically significant role were wrist guards, steepness of slope, and scene of injury (terrain park vs slopes); $96 \%$ of shoulder dislocations secondary to snowboarding were anterior glenohumeral dislocations. ${ }^{25}$ The treating physician should be alert for acute traumatic rotator-cuff tears in the setting of dislocation in older riders.

Following shoulder dislocation, immediate reduction should be performed. Management is then dictated by patient age, history of recurrent instability, and concomitant injuries. ${ }^{26}$ For a first dislocation, a short period of immobilization is recommended, followed by 4-6 weeks of rehabilitation focused on obtaining full range of motion and strength of the affected shoulder and extremity. ${ }^{26}$ In patients treated operatively, extensive postsurgical rehabilitation is recommended and varies based on the surgery performed. Return to play thus varies widely, but is generally based on full, painless range of motion and full strength. ${ }^{26}$

\section{Rotator-cuff injury}

Rotator-cuff injury tends to occur with falls forward, as do most shoulder injuries in snowboarding. ${ }^{1}$ Appropriate management ultimately depends on the extent of rotator-cuff tearing, patient age, and concomitant injuries. ${ }^{26}$ For acute, full-thickness tears, surgery is often warranted, followed by extensive physical therapy. Partial rotator-cuff tears are typically treated conservatively, with physical therapy as the focus of treatment. Physical therapy should include range of motion, strengthening, core and kinetic chain strengthening, scapular stabilization, shoulder biomechanics, high-repetition and low-resistance exercises, and specific sport-related exercises. ${ }^{26}$ Return to sport is highly variable and often dependent on the age of the patient, severity of pathology, treatment elected, and patient expectations. ${ }^{26}$

\section{Lower extremity}

Lower-extremity injuries are less common than upperextremity injuries among snowboarders, as boot and binding technology has evolved; however, lower-extremity injuries are more common among skilled snowboarders. Ishimaru et al reviewed nearly 8,000 snowboarding injuries that presented to their emergency department between the 2004-2005 and 2008-2009 seasons. ${ }^{28}$ Lower-extremity injuries accounted for $12.3 \%$ of all snowboarding injuries, with ankle injury being the most common. ${ }^{28}$ When elite snowboarders to recreational snowboarders are compared, knee injuries are responsible for the largest increase in injury incidence. ${ }^{29}$ The mechanism responsible for most lower-extremity injuries in Ishimaru et al was collision with other participants, obstacles $(38.9 \%)$, or isolated falls $(36.1 \%)$, while Kirkpatrick et al noted that falls were the most common mechanism of injury. ${ }^{28,30}$ Many lower-extremity injuries can be attributed to the type of boot worn. ${ }^{4}$ Approximately $90 \%$ of snowboarders wear soft boots, but racers and professional snowboarders often wear hard boots. ${ }^{28}$ Ankle injuries are more common among athletes who wear soft boots, and knee injuries are more common among athletes who wear hard boots. ${ }^{1}$ Lower-extremity injuries in snowboarding also range from ligament sprains to fractures, and can involve the entire lower extremity from hip to toe.

\section{Metatarsal fracture}

Kirkpatrick et al found that metatarsal fractures accounted for $88 \%$ of all foot fractures sustained by snowboarders. ${ }^{30}$ These injuries are typically secondary to an impact, rather than a rotational mechanism. A hard landing on a flat surface does not allow energy to dissipate readily, as opposed to landing on a downhill slope, and this may predispose snowboarders to more severe variants, such as Lisfranc or fifth metatarsal base fractures. As with any higher-energy mechanism, the treating physician should always have a high index of suspicion for associated injuries.

The majority of isolated metatarsal fractures can be treated initially with physical therapy that allows weightbearing as tolerated in a hard-sole shoe or walking boot. Passive and active range of motion of the unaffected joints can be started immediately, followed by strengthening exercises. Proprioception and balance may begin when tolerated, with a wobble board or balance ball. The athlete should have full, painless range of motion of the foot and ankle and be able to 
walk normally without any assistive device prior to return to sport, typically around 4-6 weeks.

Fifth metatarsal base fractures involving zones 2 and 3 are managed differently, due to the poor blood supply in this region of the bone and resultant risk for development of a nonunion. These fractures typically require a period of restricted weight-bearing or sometimes surgical intervention in elite athletes or those with significant fracture displacement. If nonoperative treatment is elected, the patient should be non-weight-bearing for a minimum of 2-6 weeks until radiographic healing, followed by 4-8 weeks of weightbearing as tolerated in a walking cast or boot. ${ }^{31}$ Return to sport is typically at 3-5 months. ${ }^{31}$ Operative fixation typically involves intramedullary screw fixation. Postoperatively, patients are kept non-weight-bearing for 2 weeks, then progressed to weight-bearing as tolerated in a walking boot until radiographic healing is demonstrated, typically around 6 weeks. ${ }^{32}$ Once radiographic healing is evident, the athlete may begin strengthening and sport-specific exercises, with return to sport at 7-12 weeks on average, depending on pain and function. ${ }^{32}$

\section{Lateral process of the talus fracture}

Fractures of the lateral process of the talus (Figure 4), also known as the "snowboarder's fracture", typically occur when a snowboarder is landing after a jump or fall, with the ankle in forced dorsiflexion and inversion. ${ }^{33}$ This fracture is often missed, and can easily be mistaken for an ankle sprain. Patients present with anterolateral ankle pain that is just anterior and inferior to the distal tip of the fibula. This fracture can be easily missed on X-ray, and delayed diagnosis can lead to avascular necrosis, nonunion, and subtalar arthritis. This injury accounts for $15 \%$ of ankle injuries in snowboarding and $34 \%$ of ankle fractures in snowboarding. ${ }^{33}$ Perera et al performed an analysis of four studies and demonstrated that $27 \%$ of these injuries were missed with a delayed diagnosis $>2$ weeks later. ${ }^{33}$ Of those patients with a delayed diagnosis, $45 \%$ had poor outcomes and persistent issues. ${ }^{33}$ Therefore, it is important to maintain high suspicion for this injury in snowboarders with anterolateral ankle pain.

For fractures of the lateral process of the talus that are very small or displaced $<2 \mathrm{~mm}$, the patient can be placed into a cast and kept non-weight-bearing for 4-6 weeks. ${ }^{34,35}$ Once radiographic healing has been demonstrated, progression to weight-bearing as tolerated and sport-specific exercises may begin. ${ }^{35}$ Return to sport occurs on average at 13 weeks. ${ }^{35}$ For fractures displaced $>2 \mathrm{~mm}$, open reduction and internal fixation or excision of small displaced fracture fragments may be indicated. ${ }^{36}$ Patients treated with excision may begin weight-bearing as tolerated immediately postoperatively. ${ }^{36}$ Patients treated with open reduction and internal fixation may begin weight-bearing when radiographic union is observed, typically anywhere from 6 to 12 weeks. ${ }^{36}$ Range of motion of unaffected joints may begin immediately postoperatively, with progressive weight-bearing and ankle range of motion beginning when radiographic healing is present. ${ }^{36}$ Once full weight-bearing and near-full range of motion is attained, the athlete may progress gradually to sport-specific exercises. Return to sport is indicated with radiographic evidence of union, painless full range of motion, and full strength, typically around 6 months. ${ }^{35}$

\section{Lateral malleolus fracture}

In Ishimaru et al, 81 of 154 (52.6\%) lower-extremity fractures involved the ankle. ${ }^{28}$ Most of these ankle fractures in snowboarders occur from a supination-external rotation mechanism resulting in a short oblique fracture of the distal fibula. Patton et al similarly showed that nearly half of all ankle injuries in snowboarders involved fractures, with the majority of the fractures affecting the lead foot. ${ }^{37}$ Appropriate management of lateral malleolus fractures depends on concomitant injuries and fracture displacement. Isolated lateral malleolus fractures with $<3 \mathrm{~mm}$ displacement and no evidence of associated syndesmotic injury may be

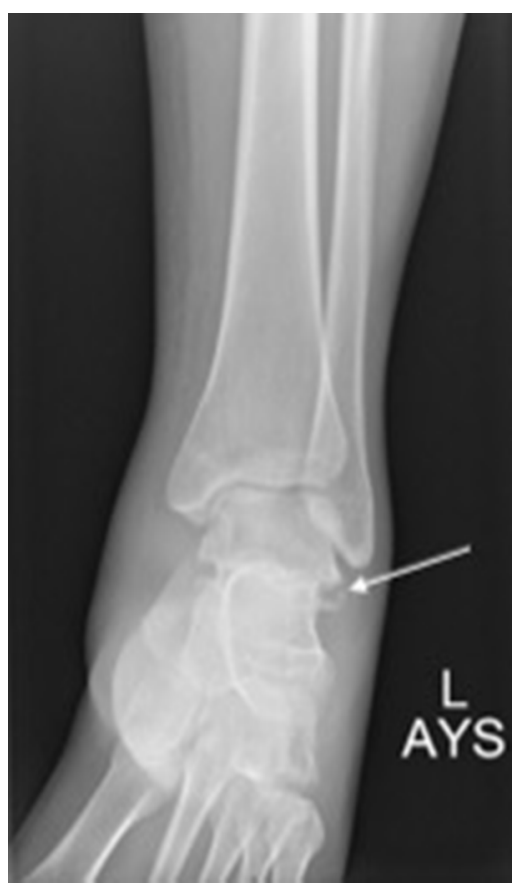

Figure 4 Lateral process of the talus fracture (white arrow). 
treated nonoperatively with a short leg cast or boot. For stable fractures, weight-bearing as tolerated in a walking boot is allowed. The patient should begin immediate range-of-motion exercises and physical therapy, focusing on ankle strengthening, stabilization, and proprioception, with progression to sport-specific exercises. The patient may return to sport once radiographic union is present, the fracture site is untender to palpation, and appropriate strength and proprioception is regained to prevent reinjury, typically around 6-8 weeks. ${ }^{38}$

If operative fixation is required, the patient should be non-weight-bearing for 2-6 weeks after surgery, depending on surgeon preference and the extent of injury. ${ }^{38}$ Dehghan et al showed no difference in early weight-bearing and late weight-bearing after operative fixation of unstable ankle fractures with regard to function scores, physical tests, wound complications, and loss of fixation or reduction. ${ }^{39}$ The athlete may return to sport when they have radiographic union, full, pain-free range of motion, and good strength with sport-specific exercises, typically achieved at a minimum of 6-12 weeks.

\section{Ankle-ligament injury}

Ankle-ligament injuries account for $46 \%-52 \%$ of all lowerextremity sprains in the snowboarding population. ${ }^{28,30}$ Although some studies have shown that soft snowboard boots place these athletes at approximately twice the risk of ankle injuries compared with hard snowboard boots, other studies have demonstrated that overall ankle-injury rates do not appear to be significantly affected by boot type. . $0,40,41^{3}$

Most ligamentous injuries to the ankle are treated nonoperatively. The athlete may begin range of motion and weightbearing as tolerated immediately, typically in a walking boot or ankle brace, depending on the severity of the sprain. At 1-2 weeks postinjury, the patient should begin proprioceptive training, strengthening, and bilateral lower-extremity closed-chain exercises. The athlete may then progress to strengthening and closed-chain exercises unilaterally on the affected leg, followed by straight-line running. At 3-4 weeks, sport-specific exercise is started in a brace for comfort. Once the patient has painless full range of motion and strength, they may return to sport, typically between 6-12 weeks, depending on injury severity. ${ }^{42}$

\section{Tibia-shaft and proximal tibia fracture}

Although more common among skiers, tibia-shaft fractures are seen in snowboarders as well. In a retrospective study of 363 skiers and snowboarders suffering tibial fractures over a 7-year period, only $8 \%$ of the tibial fractures occurred in snowboarders. ${ }^{43}$ Ishimaru et al showed that tibia-shaft fractures accounted for $42 \%$ of all lower-leg fractures in their series of snowboarding injuries. ${ }^{28}$ Similar to skiers, hard snowboard boots place snowboarders at risk for fractures of the fibula and tibia at the top of the boot, known as a boottop fracture..$^{40}$

Tibia-shaft fractures are typically treated with intramedullary nail or plate constructs. Diaphyseal tibia fractures treated with a plate construct should be non-weight-bearing or touchdown-weight-bearing initially, and slowly progress to full weight-bearing at 10-12 weeks. For tibia-shaft fractures treated with an intramedullary nail, the patient may typically begin partial weight-bearing with crutches immediately postoperatively and progress as tolerated to full weightbearing. ${ }^{38,44}$ For fractures with high levels of comminution, partial weight-bearing with crutches may be maintained until fracture callus is visible on imaging. Range of motion of the unaffected joints, as well as passive range of motion of the knee, should start immediately. Strengthening exercises may begin once there is evidence of radiographic healing and painless weight-bearing. Return to sport for diaphyseal tibia fractures is typically at 10-12 months once the athlete demonstrates healing, has painless range of motion, and is back to baseline strength. ${ }^{45}$

Tibial plateau fractures account for only about $5 \%$ of all lower-leg fractures in snowboarders. ${ }^{28}$ Stenroos et al found tibial plateau fractures slightly more likely than tibia-shaft fractures in snowboarders. ${ }^{43}$ Snowboarders were also more likely than skiers to suffer complex periarticular fractures. ${ }^{43}$ Complex proximal tibia fractures have been associated with high energy and axial pressure with rotational forces, often associated with loss of control while jumping with hard snowboard boots. ${ }^{40,46}$

Tibial plateau fractures may be treated surgically or nonoperatively, depending on fracture type, though some athletes may be treated operatively to accelerate their return to sport. Following operative fixation, the patient should be non-weight-bearing for 4-6 weeks. Patellar mobility drills, range of motion of unaffected joints, and passive range of motion of the knee with assistance from a physical therapist should start immediately. ${ }^{38}$ Weight-bearing is initiated at 6-10 weeks postoperatively, starting with partial weight-bearing and gradually weaning off crutches. The athlete may then begin low-impact exercise as tolerated and proprioceptive training. At 10-16 weeks, strengthening exercises of the bilateral lower extremities are introduced, with progression to unilateral lower-extremity strengthening exercises. At 
20-24 weeks, if the athlete has advanced without issue in physical therapy to that point, they may begin higher-impact and sport-specific exercises. ${ }^{47}$ Return to sport is typically at 7-12 months after operative fixation of a tibial plateau fracture. ${ }^{48}$ No data have been published on return to sport for nonoperatively treated tibial plateau fractures, though standard guidelines of radiographic union, full motion, normal strength, and proprioceptive control will direct sport resumption.

\section{Anterior cruciate ligament tear}

Ligamentous injuries to the knee are more commonly seen in skiers than snowboarders, due to the lower torsional forces seen in snowboarders. Unlike skiers, most binding systems in snowboarders do not release, and thus the rotatory forces about the knee are significantly reduced, preventing many anterior cruciate ligament (ACL) injuries. ${ }^{4}$ Soft boots are now the more popular boot type and have decreased the incidence of knee injuries, but elite-level athletes often still wear hard boots and perform jumps and tricks, thus increasing their risk of ACL injury. ${ }^{49}$ Ehrnthaller et al showed this trend toward more injuries about the knee in professional compared to recreational snowboarders: whereas knee-injury rates occur in about $6 \%$ of the general snowboarding population, these rates more than triple to about $20 \%$ in the elite-snowboarding population. ${ }^{50-55}$

Although ACL injuries can be treated conservatively, the majority of acute ACL injuries in athletes are treated with surgical intervention to improve knee stability. In addition, these patients should be examined carefully to avoid missing a multiple-ligament knee injury or any associated neurovascular disruption. Following ACL reconstruction, the athlete should begin patellar mobilization, knee range of motion as tolerated, and immediate weight-bearing as tolerated, with crutches for support if there are no concomitant injuries restricting weight-bearing. The patient should be weaned from crutches by 2 weeks postoperatively. At 1-4 weeks, therapy should also consist of closed-kinetic-chain quadriceps-strengthening exercises, balance and proprioceptive exercises, and stationary bicycle. Once the patient has full passive range of motion of the knee, good strength, and a normal gait, they should begin stair climbing with progression of closed-kinetic-chain, proprioceptive, balance, and strengthening exercises. At 10-16 weeks postoperatively, physical therapy should focus on continued progression of range of motion and strength, with a goal of progressing to jogging at 12 weeks. Sport-specific drills may be initiated 4-6 months after surgery. Athletes typically return to sport around 9 months, when they are pain-free and have the nec- essary range of motion, strength, and balance to participate in snowboarding.

\section{Spine}

Though rare, spine injuries can be among the most catastrophic of snowboarding injuries. ${ }^{56}$ One study evaluated patients with severe snowboarding injuries, identified as an injury-severity score $\geq 15$, and found that $22.2 \%$ of these injuries involved the spine. ${ }^{56}$ Thoracolumbar junction fracture is the most common spine injury in snowboarding, with cervical spine-compression fractures second and sacral fractures third. ${ }^{56,57}$ Spine injuries in snowboarding are most often secondary to jumps and falls, rather than collisions. Axial load is the most common mechanism, followed by flexion-distraction moment..$^{57,58}$ In a study of spine fractures sustained while skiing and snowboarding, Gertzbein et al demonstrated that snowboarders experience compression-mechanism injuries (Figure 5) and isolated transverse process and spinous process fractures, while distraction and rotation injuries occur in skiers. ${ }^{58}$

For treatment of these spine injuries, classic principles hold true. Isolated and stable compression fractures are treated nonoperatively, with bracing indicated for some injuries, depending on location and patient comfort. Burst fractures and other unstable fractures of the spine are treated

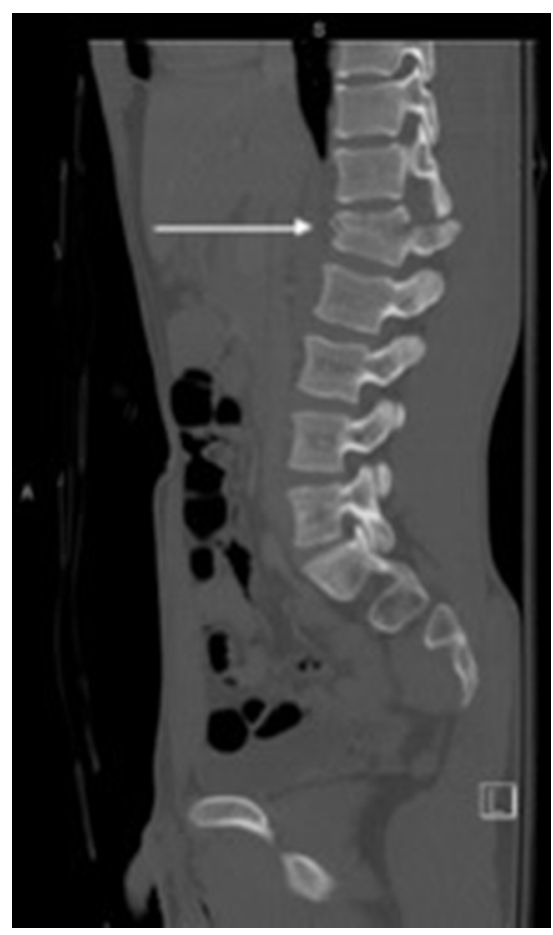

Figure 5 Lumbar spine burst fracture (white arrow). 
with surgical stabilization as indicated by their respective patterns. When spine injuries are secondary to a high-energy mechanism, such as a high-speed collision, there may be associated spinal cord injury. Rehabilitation after spine injury is determined by the level of dysfunction and need for operative or nonoperative treatment. Rehabilitation generally focuses on activities of daily living, with the goal of return to a functional standard of living.

Patients with compression fractures that do not require bracing or operative intervention may continue normal daily activity as tolerated, but should be held from sport for at least 3 months..$^{59}$ Patients should typically rest for 4-6 weeks and then begin low-impact exercises and trunk stabilization, progressing slowly to higher-impact and sport-specific drills. For compression fractures that require bracing, rehabilitation protocol and return to sport should be determined by the treating spine surgeon and should not occur prior to 3 months. ${ }^{59}$ Severe compression fractures that require operative intervention will also have varying rehabilitation protocols and return to sport dictated by the fracture type and the operating surgeon. Return to sport is contraindicated in athletes who require spinal fusion that crosses the cervicothoracic or thoracolumbar junctions or terminates at these junctions. ${ }^{60}$ If an athlete has a spinal fusion that does not cross or terminate at these points, they may return to sport once they obtain full, painless range of motion, normal strength, have no neuro- logic deficits, and no spine tenderness to palpation. ${ }^{61}$ Masuda et al reported demographic information on 19 patients who had snowboarding spine injuries, none of whom returned to sport. ${ }^{60}$ For any patients who experience transient paresis at the time of injury and have an underlying anatomic abnormality, we recommend evaluation and treatment by a spine specialist before considering return to sport.

Patients with isolated transverse process and spinous process fractures may continue daily activities as tolerated, but should refrain from return to sport until they have painless, full spine range of motion, no neurological deficits, and at least $80 \%$ muscle strength. ${ }^{62,63}$ Return to activity should begin with low-impact exercises and progress as tolerated to sport-specific activities. ${ }^{62,63}$ Return to sport typically occurs around 4-8 weeks. ${ }^{62,63}$

\section{Pelvis}

In a study evaluating snowboarding-related pelvic trauma, Ogawa et al found that pelvic fractures accounted for $2 \%$ of all fractures associated with the sport. ${ }^{64}$ Falls and hard landings from jumps are the primary mechanisms of pelvic injury. Collisions with trees and ski towers tend to be less common causes of pelvic fractures, but are associated with more unstable injury patterns. Ogawa et al noted that $85.5 \%$ of all pelvic fractures in their series were stable and that pubic and ischial fractures (Figure 6) represented the largest

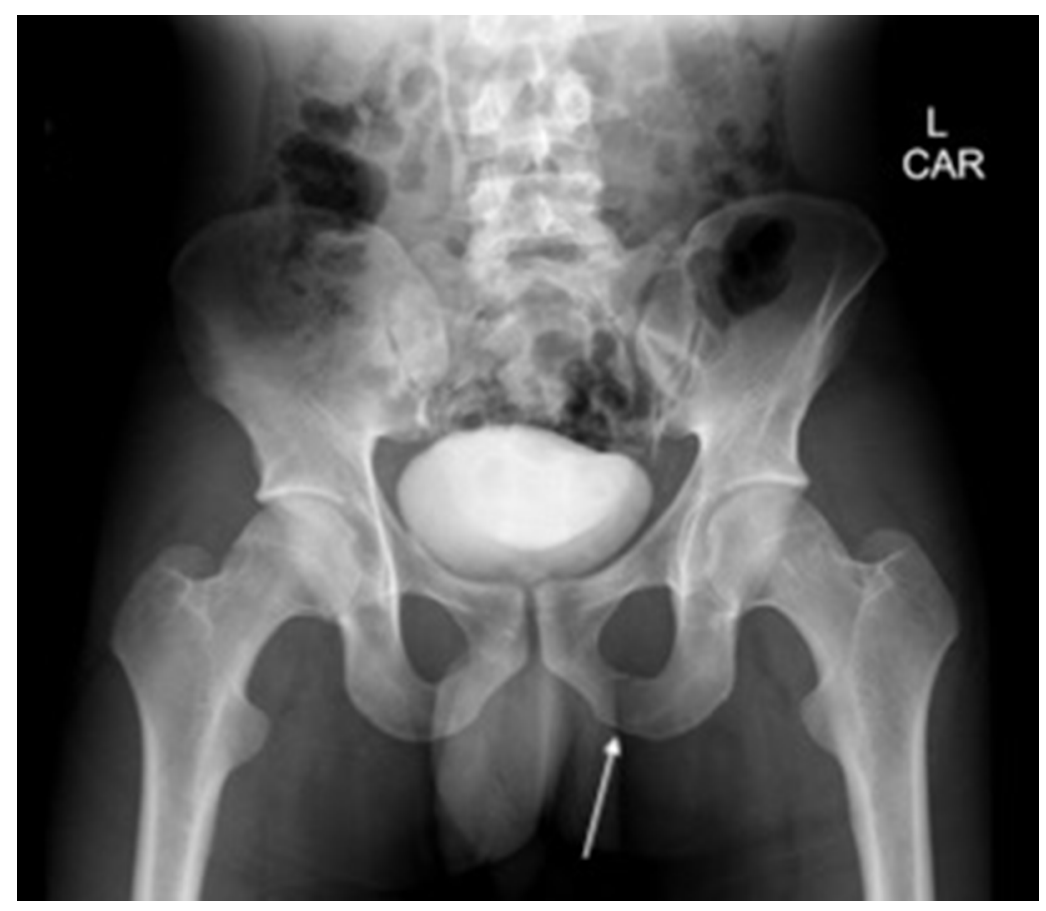

Figure 6 Isolated pubic ramus fracture (white arrow). 
percentage of all fractures, at $46.9 \%{ }^{64}$ Though isolated sacral fractures are rare, they made up $24.1 \%$ of pelvic fractures in Ogawa's series. ${ }^{64}$ Treating providers should keep this in mind with any snowboarder presenting with buttock pain after injury. Associated injuries were noted in $20 \%$ of riders with pelvic fractures, with higher incidence noted in patients with unstable pelvic fractures.

In general, isolated pubic ramus, ischium, and undisplaced sacral fractures with no neurological involvement are typically treated nonoperatively and may be weight-bearing as tolerated. Although specific rehabilitation protocols and return to snowboarding have not been described in the literature for these fractures, general principles for recovery can be followed. Range of motion and maintaining equivalent bilateral lowerextremity strength are included in the goals of rehabilitation for these injuries. The patient should be pain-free with full range of motion, full strength, and return of normal body control before return to sport. Unstable pelvic fractures are frequently treated surgically, and return to high-level activity is more prolonged and complex and may be precluded in some patterns. ${ }^{65}$ Initial activity is dictated by the fracture pattern and fixation performed. Final goals are similar to pelvic fractures treated nonoperatively, with restoration of lower-extremity function and strength guiding return.

\section{Conclusion}

Snowboarding and terrain parks have steadily increased in popularity, leading to an increase in snowboarding injuries being seen by orthopedic providers. The most common injuries seen in snowboarders are generally upper-extremity injuries. Despite many of the injuries seen in snowboarding also occurring in other sports, objective data about rehabilitation and return to sport is lacking. This provides an opportunity for future research in the area of rehabilitation and return to sport for general and sport-specific injuries. Additionally, further research is warranted in snowboardingbiomechanics studies, with the aim of prevention of injuries and development of effective protective equipment.

\section{Disclosure}

The authors report no conflicts of interest in this work.

\section{References}

1. Owens BD, Nacca C, Harris AP, Feller RJ. Comprehensive Review of Skiing and Snowboarding Injuries. J Am Acad Orthop Surg. 2018;26(1):e1-e10.

2. Kim S, Endres NK, Johnson RJ, Ettlinger CF, Shealy JE. Snowboarding injuries: Trends over time and comparisons with alpine skiing injuries. Am J Sports Med. 2012;40(4):770-776.
3. Made C, Elmqvist LG. A 10-year study of snowboard injuries in Lapland Sweden. Scand J Med Sci Sports. 2004;14(2):128-133.

4. Sachtleben TR. Snowboarding injuries. Curr Sports Med Rep. 2011;10(6):340-344.

5. Rust DA, Gilmore CJ, Treme G. Injury patterns at a large Western United States ski resort with and without snowboarders: the Taos experience. Am J Sports Med. 2013;41(3):652-656.

6. Covassin T, Elbin RJ. The cognitive effects and decrements following concussion. Open Access J Sports Med. 2010;1:55-61.

7. Strand SL. Post-concussive syndrome in a female basketball player: a case study. Open Access J Sports Med. 2013;4:123-125.

8. Idzikowski JR, Janes PC, Abbott PJ. Upper extremity snowboarding injuries. Ten-year results from the Colorado snowboard injury survey. Am J Sports Med. 2000;28(6):825-832.

9. Beleckas C, Calfee R. Distal radius fractures in the athlete. Curr Rev Musculoskelet Med. 2017;10(1):62-71.

10. Henn CM, Wolfe SW. Distal radius fractures in athletes: approaches and treatment considerations. Sports Med Arthrosc Rev. 2014;22(1):29-38.

11. Morrell NT, Moyer A, Quinlan N, Shafritz AB. Scapholunate and perilunate injuries in the athlete. Curr Rev Musculoskelet Med. 2017; 10(1):45-52.

12. Winston MJ, Weiland AJ. Scaphoid fractures in the athlete. Curr Rev Musculoskelet Med. 2017;10(1):38-44.

13. Halim A, Weiss AP. Return to Play After Hand and Wrist Fractures. Clin Sports Med. 2016;35(4):597-608.

14. Symes TH, Stothard J. A systematic review of the treatment of acute fractures of the scaphoid. J Hand Surg Eur Vol. 2011;36(9):802-810.

15. Budoff JE. Treatment of acute lunate and perilunate dislocations. $J$ Hand Surg Am. 2008;33(8):1424-1432.

16. Morrell NT, Moyer A, Quinlan N, Shafritz AB. Scapholunate and perilunate injuries in the athlete. Curr Rev Musculoskelet Med. 2017;10(1):45-52.

17. Goldfarb CA, Puri SK, Carlson MG. Diagnosis, Treatment, and Return to Play for Four Common Sports Injuries of the Hand and Wrist. J Am Acad Orthop Surg. 2016;24(12):853-862.

18. Bissell BT, Johnson RJ, Shafritz AB, Chase DC, Ettlinger CF. Epidemiology and risk factors of humerus fractures among skiers and snowboarders. Am J Sports Med. 2008;36(10):1880-1888.

19. Ahrens P, Martetschläger F, Siebenlist S, et al. Return to sports after plate fixation of humeral head fractures 65 cases with minimum 24-month follow-up. BMC Musculoskelet Disord. 2017;18(1):173.

20. Clement ND. Management of Humeral Shaft Fractures; Non-Operative Versus Operative. Arch Trauma Res. 2015;4(2):e28013.

21. Beazley JC, Baraza N, Jordan R, Modi CS. Distal Humeral FracturesCurrent Concepts. Open Orthop J. 2017;11:1353-1363.

22. Kim S, Endres NK, Johnson RJ, Ettlinger CF, Shealy JE. Snowboarding injuries: trends over time and comparisons with alpine skiing injuries. Am J Sports Med. 2012;40(4):770-776.

23. Robertson GA, Wood AM. Return to sport following clavicle fractures: a systematic review. Br Med Bull. 2016;119(1):111-128.

24. Ranalletta M, Rossi LA, Piuzzi NS, Bertona A, Bongiovanni SL, Maignon G. Return to sports after plate fixation of displaced midshaft clavicular fractures in athletes. Am J Sports Med. 2015;43(3):565-569.

25. Ogawa H, Sumi H, Sumi Y, Shimizu K. Glenohumeral dislocations in snowboarding and skiing. Injury. 2011;42(11):1241-1247.

26. Hudson VJ. Evaluation, diagnosis, and treatment of shoulder injuries in athletes. Clin Sports Med. 2010;29(1):19-32.

27. Yamauchi K, Wakahara K, Fukuta M, et al. Characteristics of upper extremity injuries sustained by falling during snowboarding: a study of 1918 cases. Am J Sports Med. 2010;38(7):1468-1474.

28. Ishimaru D, Ogawa H, Sumi H, Sumi Y, Shimizu K. Lower extremity injuries in snowboarding. J Trauma. 2011;70(3):E48-E52.

29. Wijdicks CA, Rosenbach BS, Flanagan TR, et al. Injuries in elite and recreational snowboarders. Br J Sports Med. 2014;48(1):11-17.

30. Kirkpatrick DP, Hunter RE, Janes PC, Mastrangelo J, Nicholas RA. The snowboarder's foot and ankle. Am J Sports Med. 1998;26(2):271-277. 
31. Porter DA. Fifth Metatarsal Jones Fractures in the Athlete. Foot Ankle Int. 2018;39(2):250-258.

32. Lareau CR, Hsu AR, Anderson RB. Return to Play in National Football League Players After Operative Jones Fracture Treatment. Foot Ankle Int. 2016;37(1):8-16.

33. Perera A, Baker JF, Lui DF, Stephens MM. The management and outcome of lateral process fracture of the talus. Foot Ankle Surg. 2010;16(1):15-20.

34. Shank JR, Benirschke SK, Swords MP. Treatment of Peripheral Talus Fractures. Foot Ankle Clin. 2017;22(1):181-192.

35. von Knoch F, Reckord U, von Knoch M, Sommer C. Fracture of the lateral process of the talus in snowboarders. J Bone Joint Surg Br. 2007;89(6):772-777.

36. Shakked RJ, Tejwani NC. Surgical treatment of talus fractures. Orthop Clin North Am. 2013;44(4):521-528.

37. Patton A, Bourne J, Theis JC. Patterns of lower limb fractures sustained during snowsports in Otago, New Zealand. $N Z$ Med $J$. 2010;123(1316):20-25.

38. Robertson GA, Wood AM. Fractures in sport: Optimising their management and outcome. World J Orthop. 2015;6(11):850-863.

39. Dehghan N, Mckee MD, Jenkinson RJ, et al. Early Weightbearing and Range of Motion Versus Non-Weightbearing and Immobilization After Open Reduction and Internal Fixation of Unstable Ankle Fractures: A Randomized Controlled Trial. J Orthop Trauma. 2016;30(7):345-352.

40. Young CC, Niedfeldt MW. Snowboarding injuries. Am Fam Physician. 1999;59(1):131-136, 141.

41. Bladin C, Mccrory P, Pogorzelski A. Snowboarding injuries: current trends and future directions. Sports Med. 2004;34(2):133-139.

42. Al-Mohrej OA, Al-Kenani NS. Acute ankle sprain: conservative or surgical approach? EFORT Open Rev. 2016;1(2):34 44.

43. Stenroos A, Pakarinen H, Jalkanen J, Mälkiä T, Handolin L. Tibial Fractures in Alpine Skiing and Snowboarding in Finland: A Retrospective Study on Fracture Types and Injury Mechanisms in 363 Patients. Scand J Surg. 2016;105(3):191-196.

44. Chang WR, Kapasi Z, Daisley S, Leach WJ. Tibial shaft fractures in football players. J Orthop Surg Res. 2007;2:11.

45. Robertson GA, Wood AM. Return to Sport After Tibial Shaft Fractures: A Systematic Review. Sports Health. 2016;8(4):324-330.

46. Schulak DJ, Gunn DR. Fractures of tibial plateaus. A review of the literature. Clin Orthop Relat Res. 1975;109(109):166-177.

47. Kraus TM, Martetschläger F, Müller D, et al. Return to sports activity after tibial plateau fractures: 89 cases with minimum 24-month followup. Am J Sports Med. 2012;40(12):2845-2852.

48. Robertson GAJ, Wong SJ, Wood AM. Return to sport following tibial plateau fractures: A systematic review. World J Orthop. 2017;8(7):574-587.
49. Davies H, Tietjens B, van Sterkenburg M, Mehgan A. Anterior cruciate ligament injuries in snowboarders: a quadriceps-induced injury. Knee Surg Sports Traumatol Arthrosc. 2009;17(9):1048-1051.

50. Ehrnthaller C, Kusche H, Gebhard F. Differences in injury distribution in professional and recreational snowboarding. Open Access $J$ Sports Med. 2015;6:109-119.

51. Sulheim S, Holme I, Rødven A, Ekeland A, Bahr R. Risk factors for injuries in alpine skiing, telemark skiing and snowboarding--casecontrol study. Br J Sports Med. 2011;45(16):1303-1309.

52. Flørenes TW, Nordsletten L, Heir S, Bahr R. Injuries among World Cup ski and snowboard athletes. Scand J Med Sci Sports. 2012;22(1):58-66.

53. Xiang H, Kelleher K, Shields BJ, Brown KJ, Smith GA. Skiing- and snowboarding-related injuries treated in U.S. emergency departments, 2002. J Trauma. 2005;58(1):112-118.

54. Torjussen J, Bahr R. Injuries among competitive snowboarders at the national elite level. Am J Sports Med. 2005;33(3):370-377.

55. Torjussen J, Bahr R. Injuries among elite snowboarders (FIS Snowboard World Cup). Br J Sports Med. 2006;40(3):230-234.

56. de Roulet A, Inaba K, Strumwasser A, Chouliaras K, et al. Severe injuries associated with skiing and snowboarding: A national trauma data bank study. J Trauma Acute Care Surg. 2017;82(4):781-786.

57. Yamakawa H, Murase S, Sakai H, et al. Spinal injuries in snowboarders: risk of jumping as an integral part of snowboarding. J Trauma. 2001;50(6):1101-1105.

58. Gertzbein SD, Khoury D, Bullington A, St John TA, Larson AI. Thoracic and lumbar fractures associated with skiing and snowboarding injuries according to the AO Comprehensive Classification. Am J Sports Med. 2012;40(8):1750-1754.

59. Eddy D, Congeni J, Loud K. A review of spine injuries and return to play. Clin J Sport Med. 2005;15(6):453-458.

60. Cook RW, Hsu WK. Return to Play After Lumbar Spine Surgery. Clin Sports Med. 2016;35(4):609-619.

61. Masuda T, Miyamoto K, Wakahara K, et al. Clinical Outcomes of Surgical Treatments for Traumatic Spinal Injuries due to Snowboarding. Asian Spine J. 2015;9(1):90-98.

62. Gray M, Catterson P. Multilevel lumbar transverse process fractures in a professional association football player: a case report. Oxf Med Case Reports. 2015;2015(5):288-291.

63. Nagasawa DT, Bui TT, Lagman C, et al. Isolated Transverse Process Fractures: A Systematic Analysis. World Neurosurg. 2017;100:336-341.

64. Ogawa H, Sumi H, Sumi Y, Shimizu K. Pelvic fractures resulting from snowboarding. Am J Sports Med. 2010;38(3):538-542.

65. Halawi MJ. Pelvic ring injuries: Surgical management and long-term outcomes. J Clin Orthop Trauma. 2016;7(1):1-6.
Open Access Journal of Sports Medicine

\section{Publish your work in this journal}

The Open Access Journal of Sports Medicine is an international, peer-reviewed, open access journal publishing original research, reports, reviews and commentaries on all areas of sports medicine. The journal is included on PubMed. The manuscript management system is completely online and includes a very quick and fair

\section{Dovepress}

peer-review system. Visit http://www.dovepress.com/testimonials.php to read real quotes from published authors. 